\begin{tabular}{|c|c|}
\hline Citation & $\begin{array}{l}\text { Marco Vigilante, Patrick Reynaert, (2016), } \\
\text { Analysis and Design of an E-Band Transformer-Coupled Low-Noise } \\
\text { Quadrature VCO in } 28-n m \text { CMOS } \\
\text { IEEE Transactions on Microwave Theory and Techniques, vol. 64, no. 4, pp. } \\
\text { 1122-1132, April } 2016 .\end{array}$ \\
\hline Archived version & $\begin{array}{l}\text { Author manuscript: the content is identical to the content of the published } \\
\text { paper, but without the final typesetting by the publisher }\end{array}$ \\
\hline Published version & $\begin{array}{l}\text { http://ieeexplore.ieee.org/stamp/stamp.jsp?tp=\&arnumber=7420746\&isnumbe } \\
\mathrm{r}=7445805\end{array}$ \\
\hline Journal homepage & \\
\hline Author contact & $\begin{array}{l}\text { Marco.vigilante@esat.kuleuven.be } \\
+3216320384\end{array}$ \\
\hline
\end{tabular}

(article begins on next page) 


\title{
Analysis and Design of an E-Band Transformer-Coupled Low-Noise Quadrature VCO in $28 \mathrm{~nm}$ CMOS
}

\author{
Marco Vigilante, Student Member, IEEE, and Patrick Reynaert, Senior Member, IEEE
}

\begin{abstract}
This paper presents an E-Band quadrature voltagecontrolled oscillator (QVCO) implemented in $28 \mathrm{~nm}$ CMOS. Two fundamental oscillators are coupled by means of gate-to-drain transformers to realize accurate quadrature phases and switched coupled inductors are added for tuning extension. Closed-form expressions of the oscillation frequency and the tuning extension design parameters are derived. The time-variant nature of the circuit-noise to phase-noise of the presented topology is investigated, resulting in simple design guidelines for optimal design. Based on the proposed techniques, the realized prototype is tunable over two bands of almost $5 \mathrm{GHz}$ each separated in frequency, while occupying only $0.031 \mathrm{~mm}^{2}$. The peak measured phase noise at $10 \mathrm{MHz}$ offset is $-117.7 \mathrm{dBc} / \mathrm{Hz}$ from a $72.7 \mathrm{GHz}$ carrier and $\mathbf{- 1 1 0} \mathrm{dBc} / \mathrm{Hz}$ from a $88.2 \mathrm{GHz}$ carrier and varies less than $3.5 \mathrm{~dB}$ within each band.
\end{abstract}

Index Terms-Quadrature voltage-controlled oscillator (VCO), millimeter-wave (mm-Wave), phase noise, impulse sensitivity function (ISF), transformer, wide tuning range, E-Band, CMOS.

\section{INTRODUCTION}

The ever increasing demand for higher data-rate and new applications poses unprecedented challenges for wireless systems. CMOS is the technology of choice for mass production digital and is therefore playing a key role in this revolution. Recently, the Federal Communication Commission (FCC) in USA and the European Conference of Postal and Telecommunications Administrations (CEPT) in Europe allocated two bands of $5 \mathrm{GHz}$ each from $71 \mathrm{GHz}$ to $76 \mathrm{GHz}$ and from 81 $\mathrm{GHz}$ to $86 \mathrm{GHz}$ (referred as E-Band) to enable high-speed point-to-point wireless link for fiber replacement/extension over short to medium distances [1]. CMOS direct-conversion transceivers stand out for excellent linearity, power and area efficiency also at mm-Wave [2]-[4]. However, this architecture poses serious requirements on the local oscillator (LO), demanding a phase noise (PN) of at least $-110 \mathrm{dBc} / \mathrm{Hz}$ at $10 \mathrm{MHz}$ offset [2] while providing quadrature (I/Q) outputs directly at the carrier frequency to enable a 16-QAM modulation. Automotive car radar in the frequency band that spans from $77 \mathrm{GHz}$ to $81 \mathrm{GHz}$, is another application that rises a lot of interest and would benefit from integrated low noise quadrature LO solutions [5].

This paper is an expanded version from the IEEE RFIC Symposium, Phoenix, AZ, USA, May 17-19, 2015.

The authors are with MICAS, Department of Electrical Engineering (ESAT), KU Leuven, Kasteelpark Arenberg 10, B-3001 Leuven, Belgium (email: marco.vigilante@esat.kuleuven.be, patrick.reynaert@esat.kuleuven.be)

This work was supported by Analog Devices Inc.
The quadrature $\mathrm{LO}$ is therefore one of the main bottlenecks that limit the full integration of such radios. To tackle this problem, several solutions have been proposed in literature, but not all of them are suitable for mm-Wave applications, especially when a large tuning range (TR) is required. In [6] a voltage-controlled oscillator (VCO) running at the carrier frequency $\left(f_{c}\right)$ followed by a buffer and a poly-phase filter (PPF) is proposed. The down side of this solution stands in the high insertion loss of the PPF, demanding a powerhungry buffer to drive it. In [7] an injection-locked frequency tripler (ILFM3) is proposed, requiring a VCO that runs at $f_{c} / 3$ plus a frequency multiplier by 2 , a frequency divider by 2 , two mixers and two injection-locked VCOs. For such topology a large number of on-chip inductors are needed, resulting in large silicon area and exacerbating the unwanted magnetic coupling from and to other circuits. Moreover, while solutions based on frequency multipliers shows wide tuning range, the work presented by Jani et al. in [8] points out that the improvement in terms of phase noise and power consumption is not obvious when the multiplication factor is limited to 2, 3 or 5. Another popular approach is based on coupling two identical oscillators running at $f_{c}$ to realize a fundamental quadrature VCO. To overcome the well known trade-off between phase noise and phase error of the classical parallel-coupled QVCO [9] a number of alternative techniques have been recently investigated [10]-[14]. Due to the large impact of parasitics at high frequency, a coupling mechanism that does not require extra components is desirable and is therefore adopted in this work.

In [15] we have shown that two fundamental VCOs can be coupled by means of gate-to-drain transformers and switched coupled inductors can be added for tuning extension. The prototype implemented in $28 \mathrm{~nm}$ CMOS is tunable over two bands of almost $5 \mathrm{GHz}$ each separated in frequency, while showing state-of-the-art phase noise performance and consuming silicon area of only $0.031 \mathrm{~mm}^{2}$. In this work a solid theoretical background based on the time-variant nature of circuit-noise to phase-noise conversion [16] is presented. Closed-form expressions of the oscillation frequency and tuning extension design parameters are thoughtfully derived and the effect of tank mismatch over the phase error is investigated, yielding simple design guidelines to minimize phase noise and phase error at the same time. In Section II the proposed transformer-coupled quadrature VCO is presented. The design considerations at $\mathrm{mm}$-Wave and the details of the circuit implementation in deep-scaled $28 \mathrm{~nm}$ CMOS are 


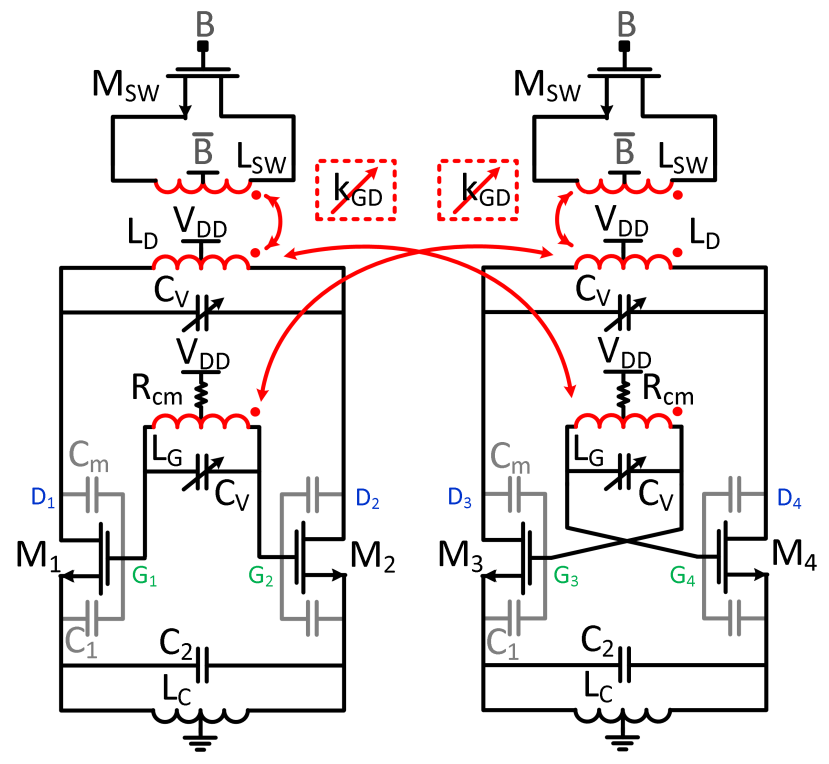

Fig. 1. Schematic of the proposed dual band quadrature VCO based on gate-to-drain transformers with switched coupled inductors.

discussed in Section III. Section IV presents the measured results, demonstrating that the prototype is tunable within two bands separated in frequency and achieves a peak phase noise at $10 \mathrm{MHz}$ offset of $-117.7 \mathrm{dBc} / \mathrm{Hz}$ from a $72.7 \mathrm{GHz}$ carrier and $-110 \mathrm{dBc} / \mathrm{Hz}$ from a $88.2 \mathrm{GHz}$ carrier and varies less than $3.5 \mathrm{~dB}$ within each band. Conclusions follow in Section V.

\section{Proposed Transformer-Coupled QuAdrature $\mathrm{VCO}$}

\section{A. Operating Principle}

Fig.1 shows the schematic of the proposed quadrature oscillator, where two VCOs based on the topology proposed in [17] are coupled by means of two gate-to-drain transformers. To prevent the circuit to oscillate in common mode, a resistor $R_{c m}$ can be added on the low current path through the center tap of $L_{G}$ as depicted in Fig.1. The LC tank at the source node is designed with a self-resonant frequency lower than the operating frequency of the VCO, it is therefore modeled as an ideal degeneration capacitor $C_{2}$ in parallel with a RF choke inductor $L_{C}$. To gain insight into the principle of operation and simplify the following analysis, it is functional to replace the resonator with its single-ended two-port admittance parameters model as in Fig.2, where $C_{D}=2 C_{V}, L_{0}=L_{G} / 2=L_{D} / 2$ and $R_{p}$ accounts for the losses. By inspection, the following equations are derived:

$$
\begin{gathered}
Y_{11}=Y_{22}=\frac{1}{R_{p}}+s C_{D}+\frac{1}{s L_{0}\left(1-k_{G D}^{2}\right)} \\
Y_{12}=Y_{21}=\frac{k_{G D}}{s L_{0}\left(1-k_{G D}^{2}\right)}
\end{gathered}
$$

In steady state due to the large signal operation, the non linearity of the active device and the pass-band behavior of the tank, it is possible to replace the transconductor with its

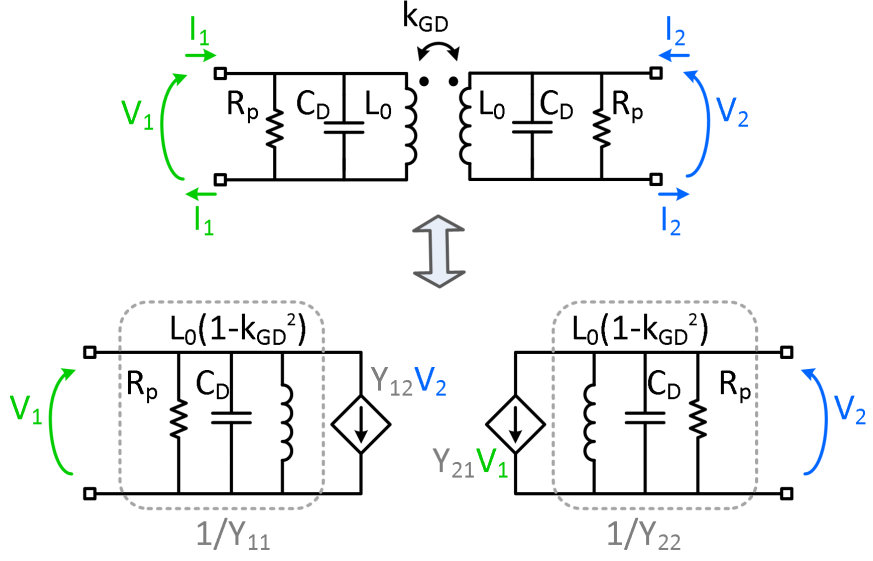

Fig. 2. Lumped element model of the proposed resonator and equivalent circuit based on the two-port admittance parameters matrix.

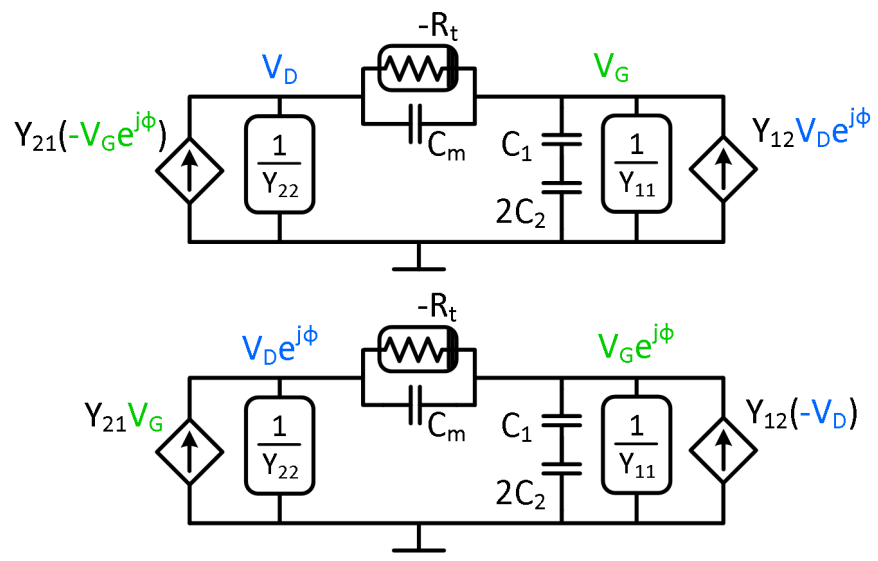

Fig. 3. Single-ended half circuit negative resistance AC model of the proposed QVCO.

describing function approximation [18]. By means of a Norton transformation and assuming differential operation (i.e. $V_{D 1}=$ $-V_{D 2}=V_{D}, V_{D 3}=-V_{D 4}=V_{D} e^{j \phi}, V_{G 1}=-V_{G 2}=V_{G}$ and $\left.V_{G 3}=-V_{G 4}=V_{G} e^{j \phi}\right)$ it is possible to redraw the circuit in Fig.1 as in Fig.3 (see Appendix for further details). From Kirchhoff's phasor nodal equations, the following expressions are derived:

$$
\begin{gathered}
V_{D}=V_{G} \cdot \frac{-Y_{21} e^{j \phi}+s C_{m}-1 / R_{t}}{Y_{22}+s C_{m}-1 / R_{t}} \\
V_{D} e^{j \phi}=V_{G} \cdot \frac{Y_{21}+e^{j \phi}\left(s C_{m}-1 / R_{t}\right)}{Y_{22}+s C_{m}-1 / R_{t}}
\end{gathered}
$$

This set of equations is verified if and only if $\phi= \pm \pi / 2$. Meaning that in presence of perfectly matched components the two oscillators are forced to operate in quadrature. Furthermore, a perfect quadrature operation is realized even if $Y_{11} \neq Y_{22}$.

\section{B. Oscillation Frequency}

To derive a closed-form expression for the oscillation frequency, it is effective to rearrange the circuit as a tank of 


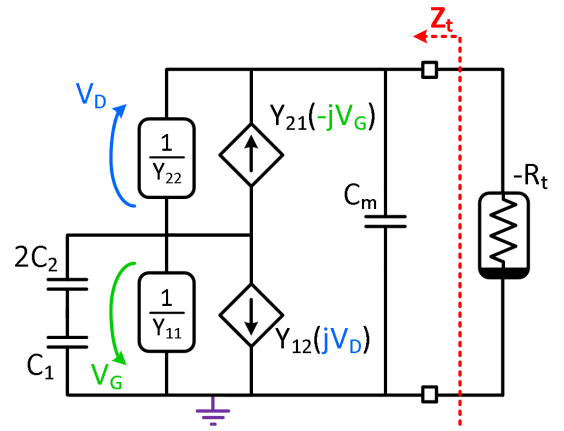

Fig. 4. Rearrangement of the circuit in Fig.3 under quadrature operation.

impedance $Z_{t}$ in parallel with an energy restoring element $-R_{t}$. By noting that the oscillator operates in quadrature, the circuit in Fig. 3 can be redrawn as shown in Fig.4. By inspection, $Z_{t}$ is derived as in (5). Assuming a high quality factor for the resonator $(R p \rightarrow+\infty)$ and imposing the condition $\operatorname{Den}\left\{Z_{t}\right\}=0$, the two resonant frequencies of the 4 th order tank are derived as

$$
\omega_{1,2}^{2}=\frac{2+\alpha_{1} \pm \sqrt{\alpha_{2}^{2}+4 k_{G D}^{2}\left(\alpha_{1}+\alpha_{3}\right)+4}}{2 L_{0} C_{m}\left(1-k_{G D}^{2}\right)\left(\alpha_{1}+\alpha_{3}\right)}
$$

where $\alpha_{1}=\left(C_{D}+C_{G}\right) / C_{m}, \alpha_{2}=\left(C_{D}-C_{G}\right) / C_{m}, \alpha_{3}=$ $C_{D} C_{G} / C_{m}^{2}$ and $C_{G}=C_{D}+C_{1} 2 C_{2} /\left(C_{1}+2 C_{2}\right)$.

Noteworthy, this expression is similar to the one derived in [12] being the two quadrature VCOs realized around similar 4th order tanks (here $C_{m}$ plays a role similar to $C_{C}$ in [12]). Moreover, in principle it is possible to use the second mode of oscillation to extend further the tuning range, provided that extra circuitry is added as already proposed for other oscillator topologies [12], [19], [20]. However, given the high target frequency of operation, in this work no effort is made to take advantage of the second resonance peak, since adding extra components would lead to higher parasitic loading of the tank. Nevertheless, during the design phase it is important to ensure that the oscillator meets the Barkhausen's criteria only in one mode. Condition easily achieved in a practical design at mm-Wave frequencies when the magnetic coupling $k_{G D}$ is designed large enough, so that $\omega_{2} \gg \omega_{1}$ and the transconductors are not able to compensate for the tank losses in the second mode.

\section{Tuning Extension}

In LC oscillators higher tuning range comes at the cost of lower spectral purity for a given power consumption. This trade-off is exacerbated at $\mathrm{mm}$-Wave, where the impact of

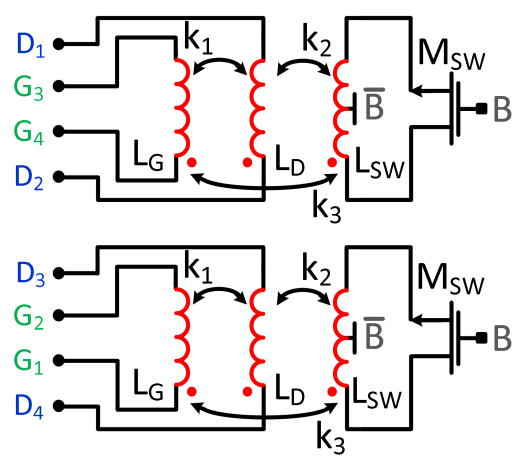

Fig. 5. Simplified lumped element model of the gate-to-drain transformers with switched coupled inductors.

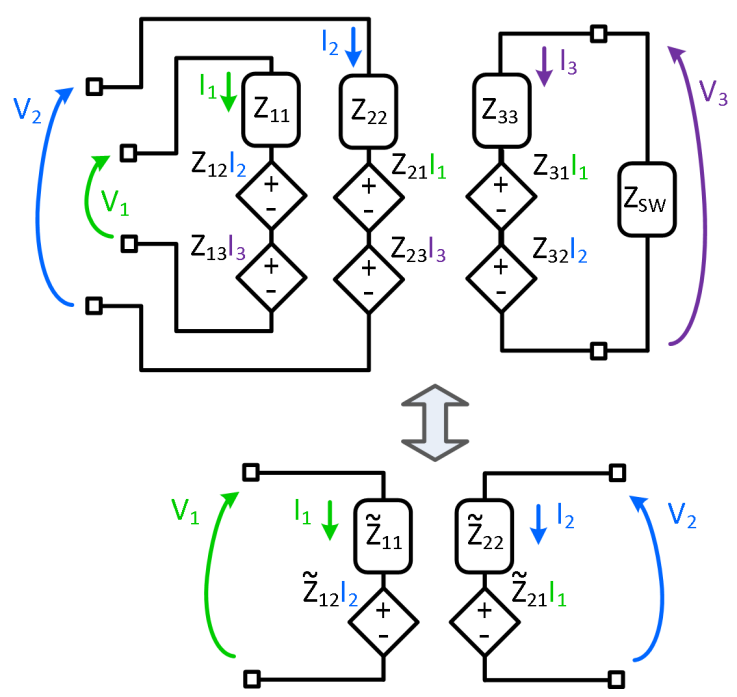

Fig. 6. Transformer with switched coupled inductor three-port impedance parameters model (top) and its two-port equivalent circuit (bottom).

parasitics is larger and the quality factor of the tank is limited by the $\mathrm{Q}$ of capacitors and varactors rather than the one of inductors. For these reasons, several recent research works have been focused on alternative tuning extension techniques [21]-[23].

From (6) it is clear that the oscillation frequency is highly sensitive to the magnetic coupling coefficient $k_{G D}$ and the inductance value $L_{0}$. In this work, a third winding $L_{S W}$ terminated on a switch $M_{S W}$ is coupled to the gate-todrain transformer to effectively change both $k_{G D}$ and $L_{0}$, as depicted in Fig.5. Intuitively, when the switch is turned $\mathrm{ON}$, the current induced in $L_{S W}$ through $k_{2}$ and $k_{3}$ finds a low impedance path. Whereas, when $M_{S W}$ is in OFF state, $L_{S W}$ is terminated on an infinite impedance and ideally no

$Z_{t}=\frac{j \omega L_{0}\left[\left(k_{G D}^{2}-1\right) L_{0} \omega^{2}+2-j \frac{2\left(k_{G D}^{2}-1\right) L_{0} \omega}{R p}\right]}{-\omega^{4} L_{0}^{2}\left(k_{G D}^{2}-1\right)\left[\left(C_{G}+C_{m}\right) C_{D}+C_{G} C_{m}\right]+j \frac{\left(k_{G D}^{2}-1\right)\left(C_{D}+C_{G}+2 C_{m}\right) L_{0}^{2} \omega^{3}}{R_{p}}+\omega^{2}\left[\frac{\left(k_{G D}^{2}-1\right) L_{0}^{2}}{R_{p}^{2}}-\left(C_{D}+C_{G}+2 C_{m}\right) L_{0}\right]+j \frac{2 L_{0} \omega}{R_{p}}+1}$ 
current is flowing. To gain deeper insight into the operation of the proposed transformer with switched coupled inductor and derive design guidelines, it is useful to refer to its three-port impedance parameter model shown in Fig.6. By inspection the following expression are derived

$$
\begin{gathered}
Z_{11}=R_{G}+s L_{G} \\
Z_{22}=R_{D}+s L_{D} \\
Z_{33}=R_{S W}+s L_{S W} \\
Z_{12}=Z_{21}=s k_{1} \sqrt{L_{G} L_{D}} \\
Z_{23}=Z_{32}=s k_{2} \sqrt{L_{D} L_{S W}} \\
Z_{13}=Z_{31}=s k_{3} \sqrt{L_{G} L_{S W}}
\end{gathered}
$$

where the losses of each inductor $\left(L_{G}, L_{D}\right.$ and $\left.L_{S W}\right)$ in Fig.5 are modelled by a series resistor (of value $R_{G}, R_{D}$ and $R_{S W}$ respectively).

Since the third port is terminated on an impedance $Z_{S W}$, it is possible to derive the equivalent two-port network as depiced in Fig.6, provided that

$$
\begin{aligned}
& \widetilde{Z}_{11}=Z_{11}-\frac{Z_{13} Z_{31}}{Z_{S W}+Z_{33}} \\
& \widetilde{Z}_{12}=Z_{12}-\frac{Z_{13} Z_{32}}{Z_{S W}+Z_{33}}
\end{aligned}
$$

where for sake of space only the expressions for the impedance and the transimpedance of the first winding are reported.

It is now possible to derive approximated equations to describe an equivalent two-port transformer. When $M_{S W}$ is in ON state $Z_{S W} \approx R_{O N}$ and assuming $R_{S W}+R_{O N} \ll \omega L_{S W}$, the equivalent series resistance and self-inductance of the primary winding $\left(R_{G, O N}, L_{G, O N}\right)$ and the equivalent magnetic coupling $k_{G D, O N}$ are

$$
\begin{gathered}
R_{G, O N} \approx R_{G}+k_{3}^{2} \frac{L_{G}}{L_{S W}}\left(R_{S W}+R_{O N}\right) \\
L_{G, O N} \approx L_{G}-L_{G} k_{3}^{2} \\
k_{G D, O N} \approx \frac{k_{1}-k_{2} k_{3}}{\sqrt{\left(1-k_{2}^{2}\right)\left(1-k_{3}^{2}\right)}}
\end{gathered}
$$

When $M_{S W}$ is in OFF state $Z_{S W} \approx 1 /\left(s C_{O F F}\right)$ and assuming $R_{S W}^{2} \ll\left(\omega L_{S W}-1 /\left(\omega C_{O F F}\right)\right)^{2}$, the following expressions are derived

$$
R_{G, O F F} \approx R_{G}+\frac{k_{3}^{2}}{\left(\omega_{S W}^{2} / \omega^{2}-1\right)^{2}} \frac{L_{G}}{L_{S W}} R_{S W}
$$

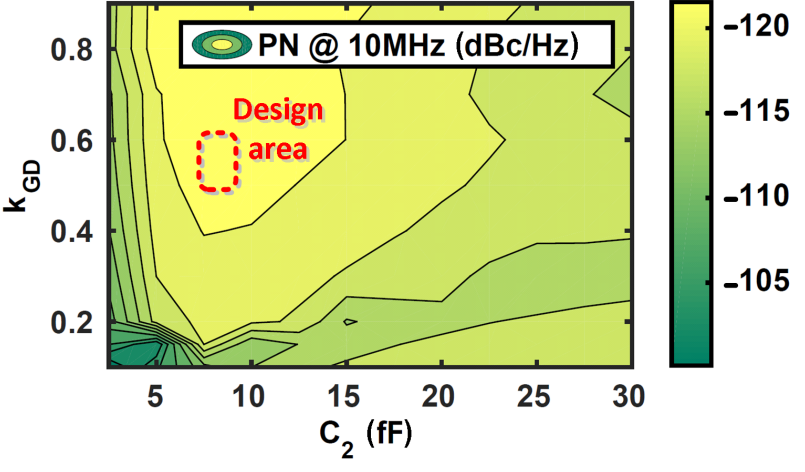

Fig. 7. Simulated phase noise at $10 \mathrm{MHz}$ offset from an $80 \mathrm{GHz}$ carrier versus $k_{G D}$ and $C_{2}$.

$$
\begin{gathered}
L_{G, O F F} \approx L_{G}+L_{G} \frac{k_{3}^{2}}{\omega_{S W}^{2} / \omega^{2}-1} \\
k_{G D, O F F} \approx \frac{k_{1}+\frac{k_{2} k_{3}}{\left(\omega_{S W}^{2} / \omega^{2}-1\right)}}{\sqrt{\left[1+\frac{k_{2}^{2}}{\omega_{S W}^{2} / \omega^{2}-1}\right]\left[1+\frac{k_{3}^{2}}{\omega_{S W}^{2} / \omega^{2}-1}\right]}}
\end{gathered}
$$

where $\omega_{S W}^{2}=1 /\left(L_{S W} C_{O F F}\right)$ is the self-resonance frequency of the third winding when terminated on the OFF capacitance of $M_{S W}$.

From (15), (16), (17), (18), (19) and (20) several design considerations can be made. Fist, the ON resistance of $M_{S W}$ severely limits the transformer losses and should be designed accordingly low enough. In addition, the current induced in the third winding effectively reduces both $L_{G, O N}$ and $k_{G D, O N}$ through $k_{2}$ and $k_{3}$.

Another critical observation deals with the design of $\omega_{S W}$ when $M_{S W}$ is in OFF state. To ensure a single solution of equation (19), $\omega_{S W}$ should be higher than the oscillation frequency $\omega_{0}$ of the VCO, imposing an upper bound for the value of $C_{O F F}$ [21], [23]. Moreover, in a practical design, the condition $\omega_{S W} \gg \omega_{0}$ is not verified. Meaning that $R_{G, O F F}, L_{G, O F F}$ and $k_{G D, O F F}$ increase with frequency and the change is sharper when $\omega_{o}$ approaches $\omega_{S W}$, demanding for a careful co-design of $M_{S W}, L_{S W}, k_{2}$ and $k_{3}$.

\section{Phase Noise}

The first step towards a design for minimum phase noise is to quantify the effect of two key design parameters (i.e. the equivalent magnetic coupling $k_{G D}$ and the degeneration capacitance $C_{2}$ ) on the operation of the proposed quadrature oscillator. Fig. 7 shows the simulated phase noise at $10 \mathrm{MHz}$ offset from an $80 \mathrm{GHz}$ carrier as a function of $k_{G D}$ and $C_{2}$. The experiments were performed adopting ideal lumped element components for passive devices where $C_{V}=10 \mathrm{fF}$, $L_{0}$ is adjusted to keep the oscillation frequency equal to $80 \mathrm{GHz}$ for fair comparison and the losses are modelled with a shunt resistor assuming a quality factor equal to 4 . The transconductors (W/L of $40 \mu \mathrm{m} / 28 \mathrm{~nm}$ ) were post-layout 

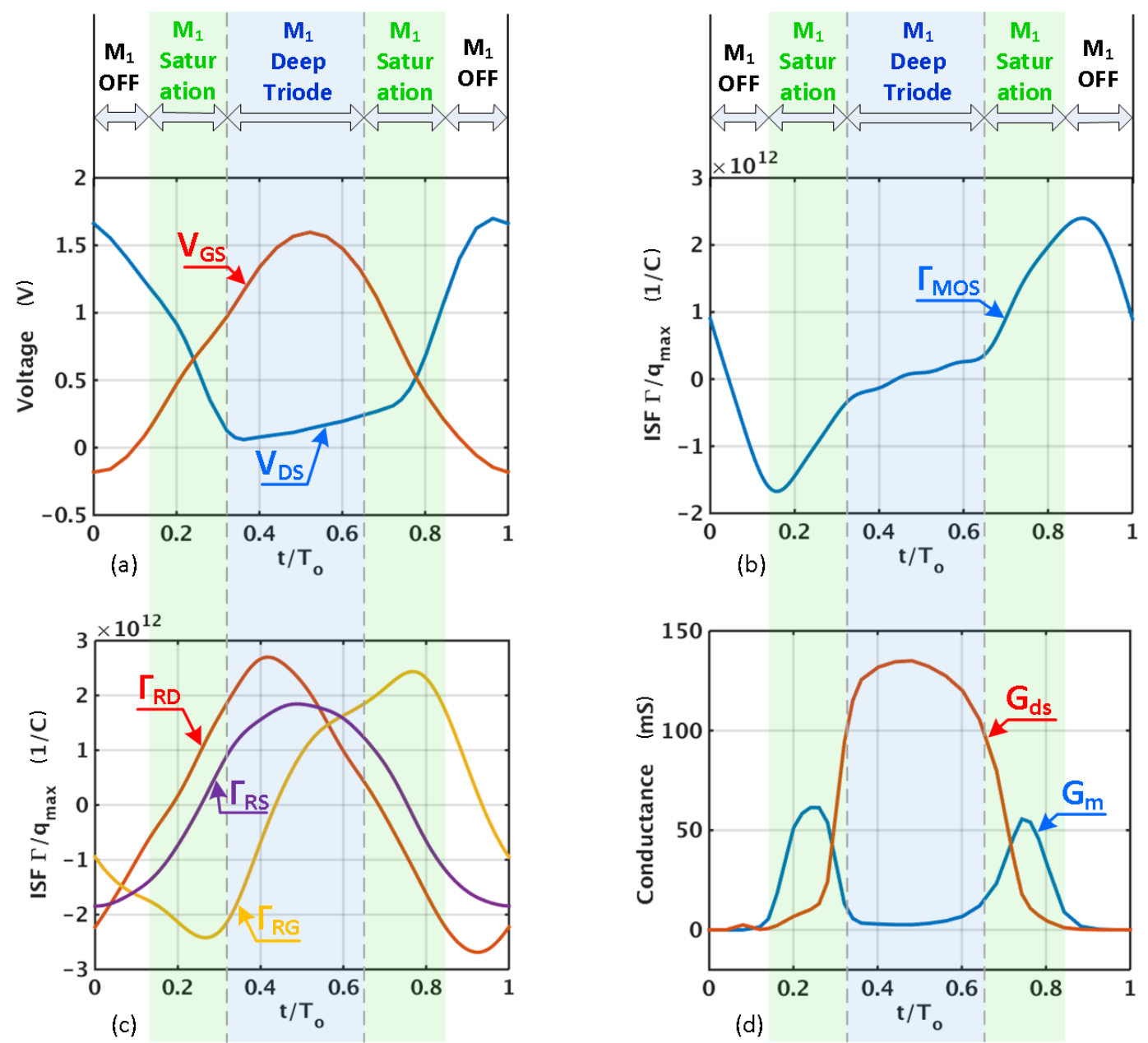

Fig. 8. Noise to phase noise conversion mechanism of the proposed oscillator. (a) Voltage waveforms and $M_{1}$ operation region. (b) Impulse sensitivity function of $M_{1}$ channel noise. (c) Impulse sensitivity function at different nodes. (d) Instantaneous transconductance $\left(G_{m}\right)$ and channel conductance $\left(G_{d s}\right)$ of $M_{1}$.

parasitic extracted to account for $C_{m}$ and $C_{1}$ (about $10 \mathrm{fF}$ and $20 \mathrm{fF}$ respectively). $R_{c m}$ is set to $100 \Omega$ to prevent oscillation in common mode. Clearly, the phase noise shows a weak dependency from $k_{G D}$, meaning that the proposed tuning extension technique can be effectively applied provided that the quality factor of the resonator is kept constant when $M_{S W}$ is in $\mathrm{ON}$ and $\mathrm{OFF}$ state. Moreover, the value of $C_{2}$ can be optimized for PN.

To get deeper insight into the circuit-noise to phase-noise conversion mechanism of the proposed topology it is useful to adopt the linear time-variant approach proposed by Hajimiri and Lee in [16]. By noting that in steady state the oscillation amplitude is limited by the compressing behavior of the transconductors, the amplitude noise is neglected and the phase noise at an angular frequency offset $\Delta \omega$ from a $\omega_{o}$ carrier can be expressed as

$$
L(\Delta \omega)=10 \log _{10}\left(\frac{\sum_{i} N_{L, i}}{2 q_{\max }^{2}(\Delta \omega)^{2}}\right)
$$

where $q_{\max }$ is the maximum charge displacement across the tank capacitor and $N_{L, i}$ is the effective noise power of the $i$-th current noise source, defined as

$$
N_{L, i}=\frac{1}{T_{o}} \int_{0}^{T_{o}} \Gamma_{i}^{2}(t) \overline{i_{n, i}^{2}(t)} d t
$$

where $\Gamma_{i}(t)$ is the impulse sensitivity function (ISF), dimensionless function of time periodic in $T_{o}=2 \pi / \omega_{o}$ and $\overline{i_{n, i}^{2}}$ is the power spectral density of the current noise produced by the $i$-th devices.

The ratio $\Gamma_{i}(t) / q_{\max }$ is accurately estimated at different oscillator nodes by means of the periodic transfer function analysis (PXF) as proposed in [24] and reported in Fig.8(c). In these experiments $k_{G D}=0.5$ and the optimum value of $C_{2}$ in Fig.7 is selected. Most importantly, Fig.8(c) shows that $\Gamma_{R S, r m s}^{2} \approx 0.65 \Gamma_{R G, r m s}^{2} \approx 0.54 \Gamma_{R D, r m s}^{2}$, meaning that the source node is the less sensitive to noise and the drain node is the most critical one. To optimize the phase noise performance is therefore important to design the transformer accordingly (i.e. the quality factor of the secondary winding $Q_{D}$ should be maximized). Moreover, the output should be probed at the source to minimize the loading effect of the following stage.

Fig.8(a) shows the voltage waveforms across $M_{1}$ highlighting its regime of operation. Due to the large voltage amplitude 


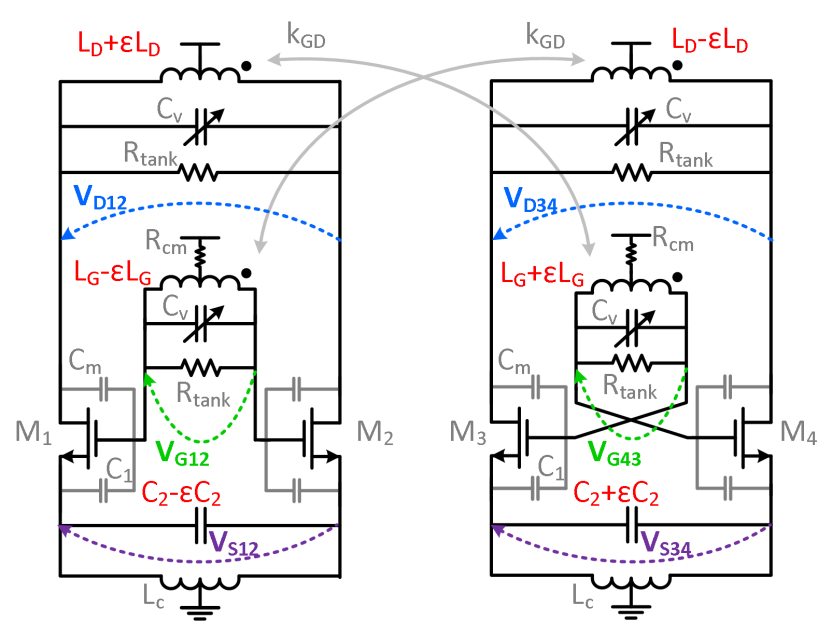

Fig. 9. Test circuit to evaluate the phase error in presence of mismatch.

the transistor operates in all three regions (i.e. saturation, triode and OFF), meaning that the active device 1) contributes to losses through the channel conductance $G_{d s}$ (referred in literature as loaded or effective Q-factor [25], [26]) and 2) injects more noise through $G_{d s}$ and the larger required $G_{m}$. Although in theory this condition is not desirable, in $\mathrm{mm}$ Wave oscillators realizing a high common mode impedance at $2 f_{o}$ over the whole tuning range is not trivial [27], and the transconductors are allowed to ender (to some extend) the triode region to maximize the voltage amplitude. The noise contribution of the transistor can be expressed as [25]

$$
\overline{i_{n, M O S}^{2}(t)}=4 K_{B} T\left(\gamma G_{m}(t)+G_{d s}(t)\right)
$$

where $K_{B}$ is the Boltzmann's constant, $T$ absolute temperature and $\gamma$ the transistor channel noise factor.

Noteworthy, Fig.8(b),(d) show that when the transistor noise contribution is at its maximum, the associated $\Gamma_{M O S}$ is close to 0 yielding a negligible associated effective noise power $N_{L, M O S} \approx 0$ during this laps of time.

\section{E. Phase Error}

In presence of mismatches in the circuit equations (3) and (4) are not valid anymore and the two oscillators depart from quadrature. This result in amplitude imbalance and phase error. The focus of this Section is the phase error, since in a practical system the LO signals are normally fed to hard limiting buffers and an I/Q mixer that is almost insensitive to small amplitude imbalance (provided that the signal amplitude is large) [9], [11]. Deriving elegant closed-form expressions for the phase error at different tank nodes under the presence of mismatches for this QVCO topology is not trivial. Moreover, the simplified linear model in Fig.3 when extended to describe mismatch due to the tank, would not account for circuit nonlinearity that may play a significant role (as is the case for QVCO that employs passive nonlinear coupler [11]).

To address this problem, it is functional to refer to the schematic depicted in Fig.9. To gain a deeper understanding, a mismatch of $2 \varepsilon=2 \%$ is imposed among passives and the

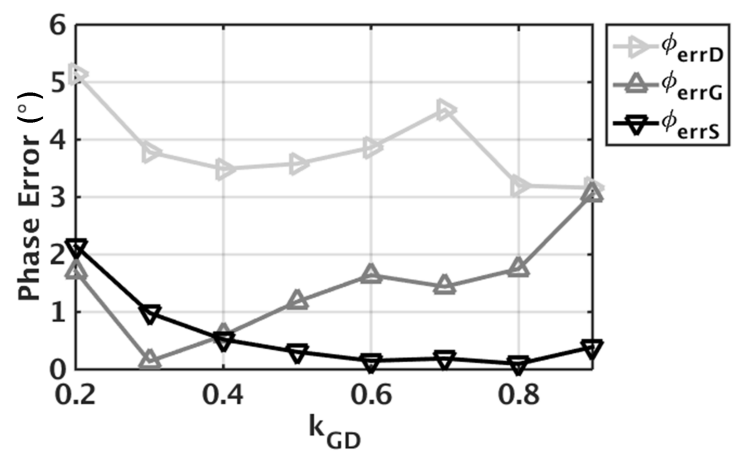

Fig. 10. Simulated impact of $2 \varepsilon=2 \%$ mismatch among passives on the phase error at different nodes against $k_{G D}$.

phase error is evaluated at different nodes. Fig.10 shows that when the equivalent magnetic coupling is $k_{G D}>0.3$ the phase error at the source node is always $\phi_{\operatorname{err} S} \leq 1^{\circ}$ and most importantly when $k_{G D}>0.4$ the condition $\phi_{\text {err } S} \leq \phi_{\text {err } G} \leq \phi_{\text {err } D}$ is achieved.

From the analysis above, we can draw two very important and perhaps unexpected conclusions, 1) probing the signal at the source leads to the same choice of optimal design parameters for both minimum phase noise (as in Fig.7) and minimum phase error (Fig.10), and 2) the loading effect of the buffer is minimal at this node (as clear from Fig.8(c) and already discussed in Section II-D).

\section{Design CONSIDERATIONS AT MM-WAVE AND CIRCUIT IMPLEMENTATION}

Thanks to the aggressive scaling of the gate length, nowadays $\mathrm{mm}$-Wave circuits can enjoy active devices with a $f_{t}$ as high as $300 \mathrm{GHz}$ in technology nodes as $28 \mathrm{~nm}$ CMOS [28]. However, when these transconductors with high intrinsic performance are used in LC oscillators the effect of the parasitics due to the layout interconnects severely limits the improvement in terms of effective $f_{t}$ and yields large fixed capacitance making the tuning range vs. phase noise trade-off tighter [29]. Furthermore, in deep-scaled CMOS processes 1) low level metals get thinner and closer to the substrate, reducing the quality factor of metal-oxide-metal (MOM) capacitors, 2) high level metals get closer to the substrate, lowering the achievable $\mathrm{Q}$ of inductors and 3) the design rule check (DRC) imposes ever increasing minimum metal density to be fulfilled in tighter area windows, limiting even further the maximum achievable Q-factor and self-resonance frequency of on-chip inductors [22], [30]. A number of design techniques are discussed in this Section to tackle the aforementioned challenges for mm-Wave LC oscillators.

As a first step, we focus on the design and layout of the active core. The parasitic gate-to-drain capacitance plays an important role in the design of any LC oscillator, lowering the oscillation frequency and limiting the tuning range [31], and the presented topology is no exception. As a matter of fact, $C_{m}$ shown in Fig.1 appears single-ended, lowering the oscillation frequency as clear from (6). This capacitance is kept minimum by adopting the transistor layout presented in [29] and shown 


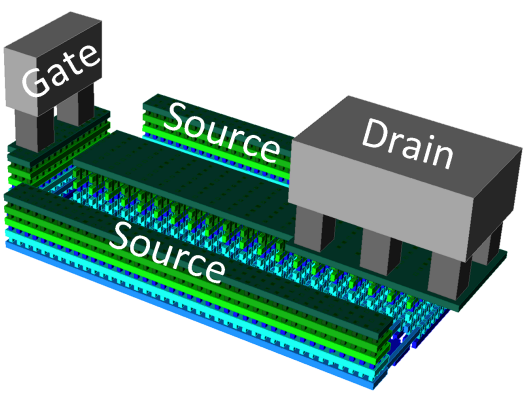

Fig. 11. 3-D layout view of the designed transistor $M_{1} 40 \mu \mathrm{m} / 28 \mathrm{~nm}$.

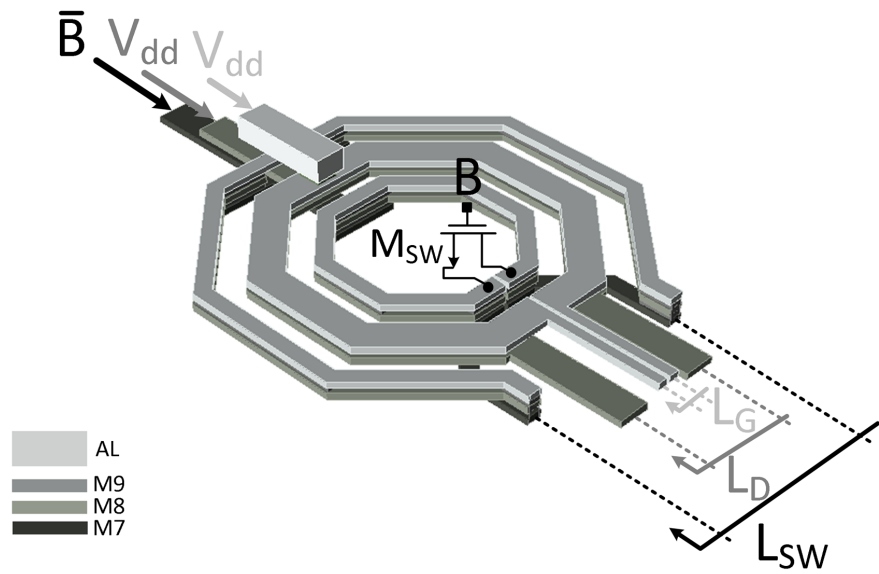

Fig. 12. 3-D layout view of the designed transformer with switched coupled inductor.

in Fig.11. Moreover, thanks to this layout, it is now possible to access directly the gate and drain of the transistor in higher metal, limiting the losses due to interconnections to the tank. The source node is accessed at both sides, minimizing the critical gain reduction due to the connection to this net and simplifying the routing to $C_{2}$ and $L_{C}$ shown in Fig.1. In this design, the trasistors are oversized to $40 \mu \mathrm{m} / 28 \mathrm{~nm}$ to account for possible model inaccuracy.

Another key aspect of any mm-Wave LC oscillator is the design and layout of the tank. Fig.12 shows the 3-D view of the layout of the proposed gate-to-drain transformer. A relatively high value of the magnetic coupling coefficient between the primary and secondary windings $k_{1}$ (see Fig.5) is desirable, so that it becomes the dominant factor in the expressions of equivalent magnetic coupling $k_{G D}$ in ON and OFF state (17), (20). Since the value of the required self-inductances is relatively low, to maximize the magnetic coupling $L_{G}$ and $L_{D}$ are realized as an overlay transformer in metal 9 and 8 respectively (see Fig.12), with a metal width of $4 \mu \mathrm{m}$ and an outer diameter of $37.8 \mu \mathrm{m}$.

The switched coupled inductor $L_{S W}$ in Fig.5 is realized with an inner coil and an outer coil in both metal 8 and 9 with $2 \mu \mathrm{m}$ width, connected together in metal 7 . The inner and outer spacing of $L_{S W}$ from the primary and secondary windings (i.e. $L_{G}$ and $L_{D}$ ) are $2.9 \mu \mathrm{m}$ and $3.5 \mu \mathrm{m}$ respectively (as shown in Fig.12). As discussed in Section II-C the value of $R_{O N}$ and $C_{O F F}$ of the switch $M_{S W}$ proves critical. Since at $\mathrm{mm}$-Wave the inductor Q-factor is relatively high, the value

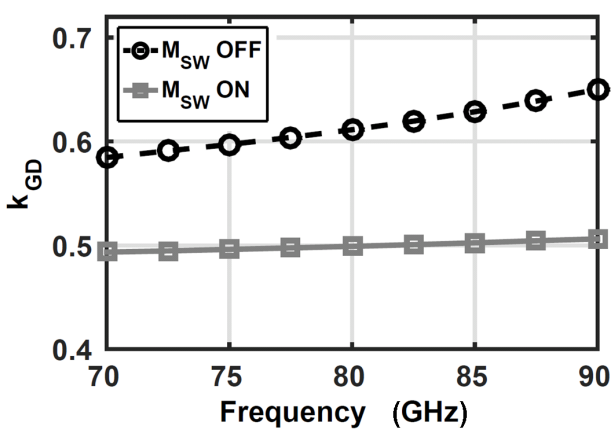

(a)

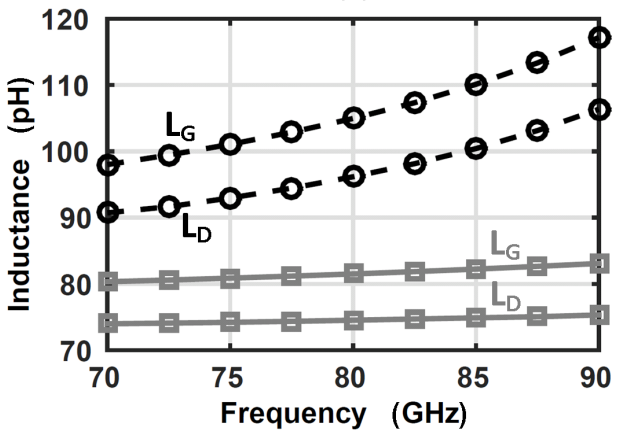

(b)

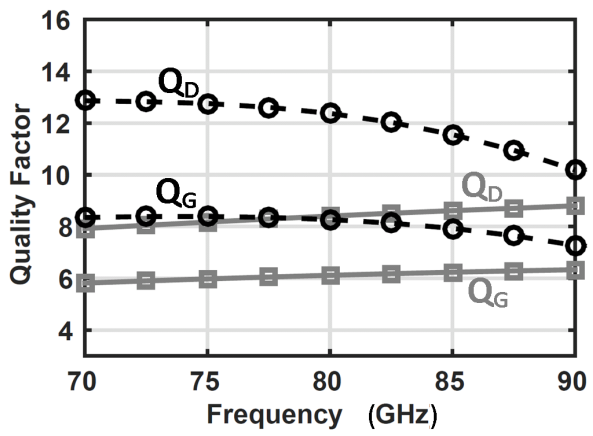

(c)

Fig. 13. Simulated characteristics of the gate-to-drain transformer with switched coupled inductor when $\mathrm{M}_{S W}$ is ON (solid grey line) and OFF (dashed black line) against frequency. (a) Equivalent magnetic coupling $k_{G D}$. (b) Self-inductance of the primary and secondary winding $\left(L_{G}\right.$ and $\left.L_{D}\right)$. (c) Quality factor of the primary and secondary winding $\left(Q_{G}\right.$ and $\left.Q_{D}\right)$.

of $R_{O N}$ will dominate the losses of the transformer in the ON state, as predicted from (15). $M_{S W}$ is therefore designed large, with a W/L of $(39 \times 3) \mu \mathrm{m} / 28 \mathrm{~nm}$. To further optimize the switch Figure of Merit $\left(\mathrm{FOM}_{S W}=R_{O N} C_{O F F}\right)$, the source and drain connections of $M_{S W}$ are layouted with a tapered via stack to minimize $C_{O F F}$. Fig.13 shows the proposed gate-todrain transformer with switched coupled inductor simulated parameters when the switch is in $\mathrm{ON}$ and OFF state. From electromagnetic simulation the equivalent magnetic coupling coefficient (Fig.13(a)), self-inductances (Fig.13(b)) and quality factors (Fig.13(c)) of primary and secondary windings of the transformer when $M_{S W}$ is $\mathrm{OFF}(\mathrm{ON})$ are $k_{G D}=0.59(0.5)$, $L_{G}=100 \mathrm{pH}(82 \mathrm{pH}), L_{D}=92 \mathrm{pH}(75 \mathrm{pH}), Q_{G}=8(6), Q_{D}=13$ (9) at $73.5 \mathrm{GHz}(83.5 \mathrm{GHz})$. It is worth to mention that, as clear from the discussion about circuit-noise to phasenoise conversion in Section II-D and shown in Fig.13(c), the winding with the higher quality factor is reserved to $L_{D}$. 


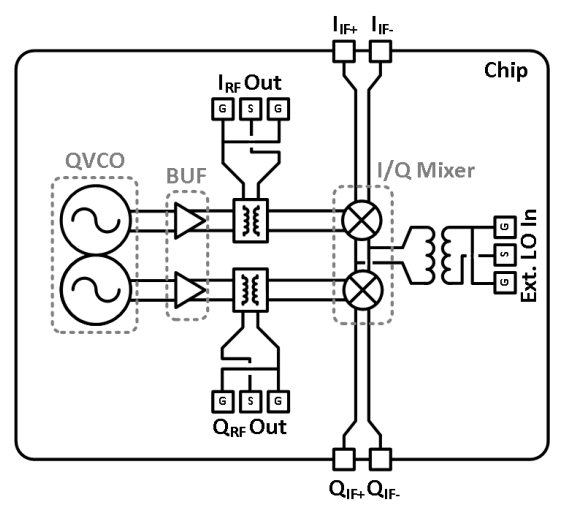

Fig. 14. Block diagram of the realized test chip.

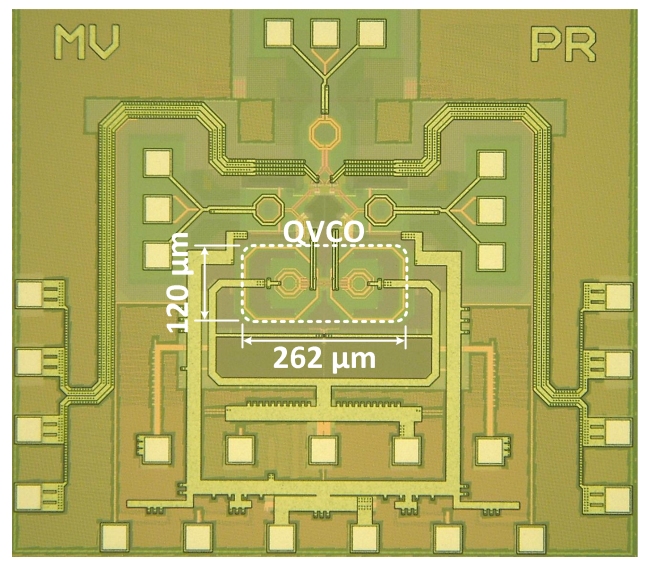

Fig. 15. Die micrograph of the realized test chip (core dimensions: $120 \mu \mathrm{m}$ x $262 \mu \mathrm{m})$.

To compensate for the degradation of the tank $\mathrm{Q}$ in the higher band (i.e. when $M_{S W}$ is in ON state), in this work the value of the degeneration capacitance $C_{2}$ is designed for optimal phase noise in this mode of operation, aiming at an uniform noise FOM over the whole tuning range. To further tune the oscillator continuously within the two bands, 2 binary-weighted digitally controlled MOM capacitors and an accumulation-mode MOS (A-MOS) varactor are added to the tank.

To minimize the flicker noise to phase noise up-conversion, a voltage-biased topology is adopted in this design. Removing the current control is a critical choice, common to several state-of-the-art low-noise mm-Wave LC oscillators (such as [13], [22], [27], [32]). In fact, the lack of ideal current sources is exacerbated at high frequencies by the larger effect of the parasitic capacitance to the substrate.

Fig.14 shows the block diagram of the realized chip. For measurement purpose, two buffers and an I/Q double-balanced mixer are also implemented on-chip. The buffers are realized with pseudo-differential neutralized common source amplifiers, providing high input-output isolation, driving the $50 \Omega$ measurement equipment directly at $\mathrm{mm}$-Wave and controlling the on-chip mixer. The latter is based on a Gilbert cell, allowing the downconversion of the high frequency on-chip quadrature signals to an intermediate frequency, instrumental to measure I/Q amplitude and phase imbalance.

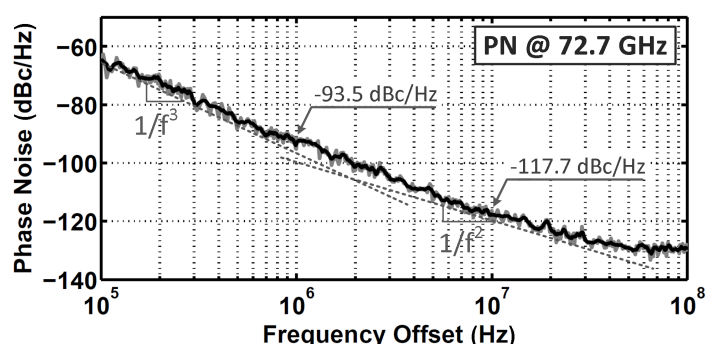

(a)

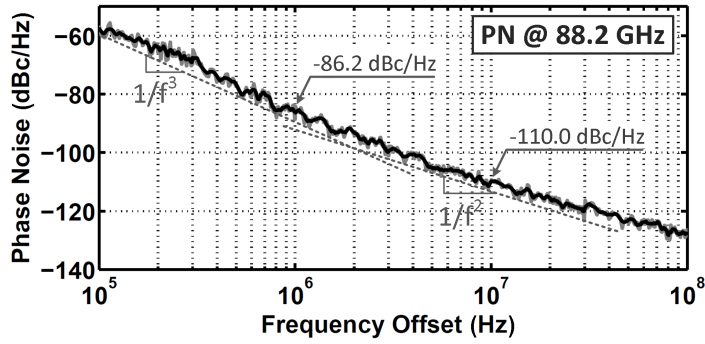

(b)

Fig. 16. Measured phase noise from a $72.7 \mathrm{GHz}$ carrier (a) and from a 88.2 $\mathrm{GHz}$ carrier (b)

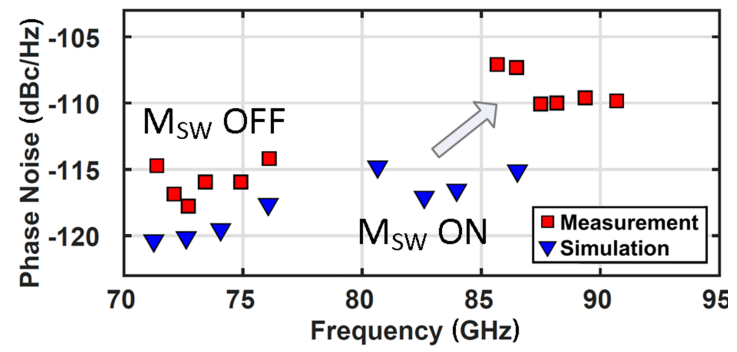

(a)

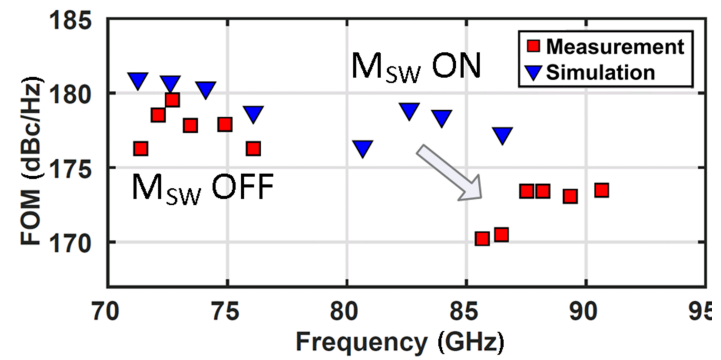

(b)

Fig. 17. Measured and simulated phase noise (a) and noise FOM (b) at $10 \mathrm{MHz}$ offset from the carrier against frequency.

\section{Measurement Results}

Fig. 15 shows the die micrograph of the quadrature VCO prototype fabricated in $28 \mathrm{~nm}$ bulk CMOS technology with no RF thick metal option. It occupies an active area of only 0.031 $\mathrm{mm}^{2}$. All the measurements are performed on a high frequency probe station. The mm-Wave output of the QVCO after the buffer and the external LO input of the double balanced I/Q mixer (see Fig.14) are directly accessed by GSG probes, while the dc and IF signal pads are wire-bonded to a printed circuit board (PCB). The quadrature VCO consumes $35.6 \mathrm{~mW}$ from a $0.7 \mathrm{~V}$ supply. The oscillation frequency is tunable from 71.4 $\mathrm{GHz}$ to $76.1 \mathrm{GHz}$ when $M_{S W}$ is OFF and from $85.6 \mathrm{GHz}$ to $90.7 \mathrm{GHz}$ when $M_{S W}$ is $\mathrm{ON}$, corresponding to $9.8 \mathrm{GHz}$ 
TABLE I

COMPARISON With STATE-OF-THE-ART INTEGRATEd QuAdRATURE Frequency GENERATION Circuits IN THE 70/100 GHz BAND

\begin{tabular}{|c|c|c|c|c|c|c|c|c|c|c|}
\hline & Topology & $\begin{array}{c}\text { Frequency } \\
(\mathbf{G H z})\end{array}$ & $\begin{array}{c}\text { TR } \\
(\mathbf{G H z})\end{array}$ & $\begin{array}{l}\text { Power } \\
(\mathrm{mW})\end{array}$ & $\begin{array}{c}\text { PN@10 MHz } \\
(\mathrm{dBc} / \mathrm{Hz})\end{array}$ & $\begin{array}{c}\text { FOM } \\
(\mathrm{dBc} / \mathrm{Hz})\end{array}$ & $\begin{array}{c}\mathbf{F O M}_{A} \\
(\mathbf{d B c} / \mathbf{H z})\end{array}$ & $\begin{array}{l}\text { Phase } \\
\text { Error }\end{array}$ & $\begin{array}{l}\text { Area } \\
\left(\mathrm{mm}^{2}\right)\end{array}$ & Technology \\
\hline$[6]$ & $\mathrm{VCO}+\mathrm{RC} \mathrm{PPF}$ & $70-89$ & 19 & 310.2 & $-107 /-114^{(1)}$ & $159 / 168.1$ & $168.7 / 177.8$ & $<8.5^{\circ}$ & $0.107^{(2)}$ & $0.35 \mu \mathrm{m} \mathrm{SiGe}$ \\
\hline [7] & ILFM3 & $70.5-85.5$ & 15 & 47.3 & $-111.7 /-115.8^{(1)}$ & $173.5 / 176.3$ & $178.9 / 181.7$ & $<2^{\circ}$ & $0.291^{(2)}$ & $65 \mathrm{~nm} \mathrm{CMOS}$ \\
\hline$[33]$ & QVCO & $90-94$ & 4 & 43.2 & $-110.5^{(1)}$ & 173.5 & n.a. & n.a. & n.a. & $65 \mathrm{~nm} \mathrm{CMOS}$ \\
\hline This & QVCO & 71.4-76.1 & 9.8 & 35.6 & $-114.2 /-117.7$ & 176.3/179.4 & 191.4/194.5 & $<1.5^{\circ}$ & 0.031 & $28 \mathrm{~nm} \mathrm{CMOS}$ \\
\hline work & & $85.6-90.7$ & & & $-107 /-110$ & $170.2 / 173.4$ & $185.3 / 188.5$ & $<3.5^{\circ}$ & & \\
\hline
\end{tabular}

(1) Estimated from the reported PN @1 MHz offset. ${ }^{(2)}$ Graphically estimated.

of total tuning range. By varying the A-MOS varactor voltage from $0 \mathrm{~V}$ to $1.2 \mathrm{~V}$ and acting on the 2 binary-weighted digitally controlled MOM capacitors, the oscillator realizes continuous tuning within the two bands.

Fig.16(a) and Fig.16(b) show the measured phase noise from a $72.7 \mathrm{GHz}$ and $88.2 \mathrm{GHz}$ carrier respectively. The signal is measured at the output of the buffer directly at $\mathrm{mm}$-Wave and downconverted with an external mixer. The prototype achieves a measured phase noise at $1 \mathrm{MHz}$ and $10 \mathrm{MHz}$ offset of $-93.5 \mathrm{dBc} / \mathrm{Hz}$ and $-117.7 \mathrm{dBc} / \mathrm{Hz}$ from a 72.7 $\mathrm{GHz}$ carrier and $-86.2 \mathrm{dBc} / \mathrm{Hz}$ and $-110 \mathrm{dBc} / \mathrm{Hz}$ from a $88.2 \mathrm{GHz}$ carrier. The measured $1 / f^{3}$ corner is $\sim 2 \mathrm{MHz}$. The same measurements are repeated over the tuning range are summarized and compared against simulations in Fig.17(a) at $10 \mathrm{MHz}$ offset, showing that the measured phase noise ranges from $-114.2 \mathrm{dBc} / \mathrm{Hz}$ to $-117.7 \mathrm{dBc} / \mathrm{Hz}$ in the lower band and from $-107 \mathrm{dBc} / \mathrm{Hz}$ to $-110 \mathrm{dBc} / \mathrm{Hz}$ in the higher one. The resulting measured noise figure of merit ranges from $176.3 \mathrm{dBc} / \mathrm{Hz}$ to $179.4 \mathrm{dBc} / \mathrm{Hz}$ and from $170.2 \mathrm{dBc} / \mathrm{Hz}$ to $173.4 \mathrm{dBc} / \mathrm{Hz}$ in the higher and lower band respectively, as reported in Fig.17(b) together with the expected results. No RF transistor model was available during the design phase, resulting in an inaccurate estimation of the $\mathrm{ON}$ resistance of $M_{S W}$, much larger than the other transistors by design as explained in Section III. The measured oscillation frequency in the higher band shows therefore a shift of about $4.6 \mathrm{GHz}$ toward higher frequencies, giving rise to a deviation from the optimal design point and a degradation of phase noise performance in this band.

To measure the quadrature amplitude and phase imbalance, an external $\mathrm{mm}$-Wave signal is applied to the on-chip I/Q mixer driven by the QVCO. The resulting downconverted IF outputs are then measured with a sampling oscilloscope and shown in Fig.18. Measurements repeated over the whole tuning range prove a phase error less than $1.5^{\circ}$ in the lower band and less than $3.5^{\circ}$ in the higher one. The amplitude error stays always below $1 \mathrm{~dB}$ in both bands. Noteworthy, in a practical system relatively simple on-chip calibration techniques may be adopted to compensate for such a limited phase error, allowing high order modulation schemes as 64QAM [4].

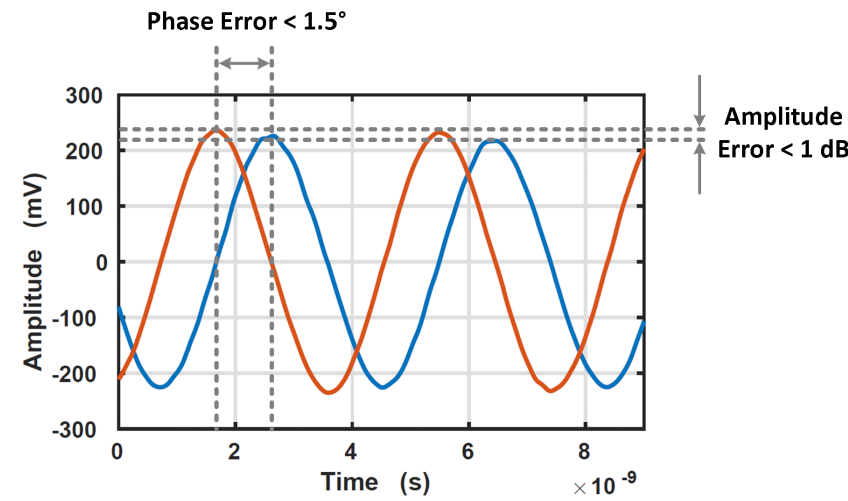

Fig. 18. Measured phase and amplitude imbalance of the I/Q signals downconverted to $260 \mathrm{MHz}$.

Table I summarizes and compares the measured performance of the quadrature VCO prototype to state-of-the-art integrated quadrature frequency generation circuits in the 70 $\mathrm{GHz}$ to $100 \mathrm{GHz}$ band. Benefited by the presented design techniques, this work achieves the lowerst power consumption while occupying the smaller silicon area, and showing a better or comparable phase noise that varies less than $3.5 \mathrm{~dB}$ within each band. To compare different designs, we adopt two noise figure of merits defined as [11], [34]

$$
\begin{gathered}
F O M=10 \log _{10}\left[\left(\frac{f_{o}}{\Delta f}\right)^{2} \frac{1}{P N(\Delta f) P_{D C}}\right] \\
F O M_{A}=10 \log _{10}\left[\left(\frac{f_{o}}{\Delta f}\right)^{2} \frac{1}{P N(\Delta f) P_{D C} \text { Area }}\right]
\end{gathered}
$$

where $f_{o}$ is the oscillation frequency, $\Delta f$ is the frequency offset from the carrier, $P_{D C}$ is the dc power consumption expressed in $\mathrm{mW}$ and Area is expressed in $\mathrm{mm}^{2}$.

When such quadrature generation circuits are employed in direct conversion transceivers, the LO feedthrough and PA pulling may become serious issues [4], [35]-[37]. It is therefore desirable to keep the number of on-chip inductors 
as small as possible and, in mm-Wave CMOS design, area serves as straightforward measure of this. Among the excellent designs in Table I, this work stands out for the lowest reported silicon area, without trading in power consumption or phase noise performance, leading to a measured $\mathrm{FOM}_{A}$ between 3.6 $\mathrm{dB}$ and $12.8 \mathrm{~dB}$ higher than the best previously reported one.

\section{CONCLUSiON}

A fundamental E-Band quadrature $\mathrm{VCO}$ based on gateto-drain transformers to realize accurate quadrature phases and switched coupled inductors for tuning extension has been presented and analyzed. An in depth discussion of the phase noise mechanism, adopted tuning extension technique and phase error under tank mismatch has been reported, leading to optimal design for minimum phase noise and minimum phase error at the same time. Layout and implementation details relevant for mm-Wave oscillators implemented in deep-scaled CMOS technology have been addressed. The QVCO prototype is tunable from $71.4 \mathrm{GHz}$ to $76.1 \mathrm{GHz}$ in the lower band and from $85.6 \mathrm{GHz}$ to $90.7 \mathrm{GHz}$ in the higher one, and shows low measured phase noise performance without trading in silicon area. The resulting $\mathrm{FOM}_{A}$ across the tuning range advances the state-of-the-art between by $3.6 \mathrm{~dB}$ and $12.8 \mathrm{~dB}$.

\section{APPENDIX}

Assuming differential quadrature operation, the equivalent ac large signal model of the circuit schematic in Fig.1 can be redrawn as in Fig.19, where the ground reference is instrumentally shifted. Following the dissertation presented by Bevilacqua and Andreani in [38], in steady state is now possible to replace the transconductor with its describing function approximation [18]. The resulting circuit is shown in Fig.20. By applying the Norton's theorem the current source $I_{\omega 0}$ can be substituted with an equivalent current source $\beta I_{\omega 0}$ in parallel with $C_{m}$ as in Fig.4, given

$$
\beta=\frac{\left(s C_{1}-j Y_{12}+Y_{22}\right) C_{2}+C_{1} Y_{22}}{\left(s C_{1}+2 Y_{22}\right) C_{2}+C_{1} Y_{22}}
$$

Since $\beta$ is in general a complex number (i.e. the current flowing through the transistor $I_{\omega 0}$ and $\beta I_{\omega 0}$ are not in phase) and the transistor does enter the triode region as discussed in Session II-D, the general result on phase noise stated in [39] and [25] does not apply. Therefore, in this work this simplified model is only used to get insight into the quadrature operation of the circuit and obtain an approximated expression of the oscillation frequency.

\section{ACKNOWLEDGMENT}

This work was supported by Analog Devices Inc, Limerick, Ireland. The authors are grateful to Mike Keaveney from Analog Devices.

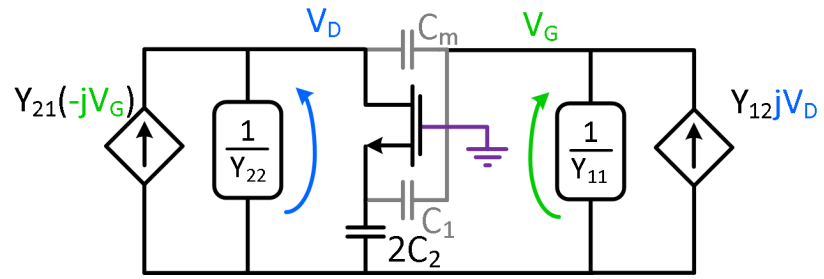

Fig. 19. Single ended AC large signal equivalent half circuit of the oscillator with an instrumental shift of the reference ground plane.

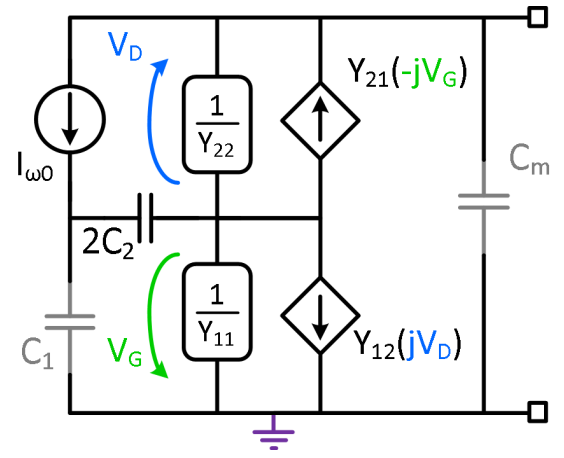

Fig. 20. Rearrangement of the circuit in Fig.19 where the transconductor is replaced with its describing function approximation.

\section{REFERENCES}

[1] J. Wells, Multigigabit Microwave and Millimeter-Wave Wireless Communications. Norwood, MA, USA: Artech House, 2010.

[2] K. Okada, K. Kondou, M. Miyahara, M. Shinagawa, H. Asada, R. Minami, T. Yamaguchi, A. Musa, Y. Tsukui, Y. Asakura, S. Tamonoki, H. Yamagishi, Y. Hino, T. Sato, H. Sakaguchi, N. Shimasaki, T. Ito, Y. Takeuchi, N. Li, Q. Bu, R. Murakami, K. Bunsen, K. Matsushita, M. Noda, and A. Matsuzawa, "Full Four-Channel $6.3-\mathrm{Gb} / \mathrm{s} 60-\mathrm{GHz}$ CMOS Transceiver With Low-Power Analog and Digital Baseband Circuitry," IEEE J. Solid-State Circuits, vol. 48, no. 1, pp. 46-65, Jan 2013.

[3] H. Wu, N.-Y. Wang, Y. Du, and M.-C. Chang, "A Blocker-Tolerant Current Mode 60-GHz Receiver With 7.5-GHz Bandwidth and 3.8-dB Minimum NF in 65-nm CMOS," IEEE Trans. Microw. Theory Techn., vol. 63, no. 3, pp. 1053-1062, March 2015.

[4] D. Zhao and P. Reynaert, "A $40 \mathrm{~nm}$ cmos e-band transmitter with compact and symmetrical layout floor-plans," IEEE J. Solid-State Circuits, vol. 50, no. 11, pp. 2560-2571, Nov 2015.

[5] S. Y. Kim, O. Inac, C.-Y. Kim, D. Shin, and G. Rebeiz, "A $76-84-\mathrm{GHz}$ 16-Element Phased-Array Receiver With a Chip-Level Built-In Self-Test System," IEEE Trans. Microw. Theory Techn., vol. 61, no. 8, pp. 30833098, Aug 2013.

[6] I. Nasr, B. Laemmle, K. Aufinger, G. Fischer, R. Weigel, and D. Kissinger, "A 70-90-GHz High-Linearity Multi-Band Quadrature Receiver in $0.35 \mu \mathrm{m} \mathrm{SiGe} \mathrm{Technology,"} \mathrm{IEEE} \mathrm{Trans.} \mathrm{Microw.} \mathrm{Theory}$ Techn., vol. 61, no. 12, pp. 4600-4612, Dec 2013.

[7] Z. Huang, H. Luong, B. Chi, Z. Wang, and H. Jia, "A 70.5-to-85.5GHz $65 \mathrm{~nm}$ phase-locked loop with passive scaling of loop filter," in Int. SolidState Circuits Conf. Tech. Dig., Feb 2015, pp. 448-450.

[8] C. Jany, A. Siligaris, J. Gonzalez-Jimenez, P. Vincent, and P. Ferrari, "A programmable frequency multiplier-by-29 architecture for millimeter wave applications," IEEE J. Solid-State Circuits, vol. PP, no. 99, pp. 1$11,2015$.

[9] A. Mazzanti, F. Svelto, and P. Andreani, "On the amplitude and phase errors of quadrature LC-tank CMOS oscillators," IEEE J. Solid-State Circuits, vol. 41, no. 6, pp. 1305-1313, June 2006.

[10] X. Yi, C. C. Boon, H. Liu, J. F. Lin, and W. M. Lim, "A 57.9-to-68.3 $\mathrm{GHz} 24.6 \mathrm{~mW}$ Frequency Synthesizer With In-Phase Injection-Coupled QVCO in $65 \mathrm{~nm}$ CMOS Technology," IEEE J. Solid-State Circuits, vol. 49, no. 2, pp. 347-359, Feb 2014.

[11] N.-C. Kuo, J.-C. Chien, and A. Niknejad, "Design and Analysis on Bidirectionally and Passively Coupled QVCO With Nonlinear Coupler," 
IEEE Trans. Microw. Theory Techn., vol. 63, no. 4, pp. 1130-1141, April 2015.

[12] M. Bajestan, V. Rezaei, and K. Entesari, "A Low Phase-Noise Wide Tuning-Range Quadrature Oscillator Using a Transformer-Based DualResonance LC Ring," IEEE Trans. Microw. Theory Techn., vol. 63, no. 4, pp. 1142-1153, April 2015.

[13] U. Decanis, A. Ghilioni, E. Monaco, A. Mazzanti, and F. Svelto, "A Low-Noise Quadrature VCO Based on Magnetically Coupled Resonators and a Wideband Frequency Divider at Millimeter Waves," IEEE J. Solid-State Circuits, vol. 46, no. 12, pp. 2943-2955, Dec 2011.

[14] M. Vigilante and P. Reynaert, "An E-Band low-noise TransformerCoupled Quadrature VCO in $40 \mathrm{~nm}$ CMOS," in European Solid-State Circuits Conf., Sept 2014, pp. 423-426.

[15] — " "A Dual-Band E-Band Quadrature VCO with Switched Coupled Transformers in 28nm HPM bulk CMOS," in IEEE Radio Freq. Integr. Circuits Symp. Dig., May 2015, pp. 119-122.

[16] A. Hajimiri and T. Lee, "A general theory of phase noise in electrical oscillators," IEEE J. Solid-State Circuits, vol. 33, no. 2, pp. 179-194, Feb 1998.

[17] L. Li, P. Reynaert, and M. Steyaert, "A Colpitts LC VCO with Millercapacitance gm enhancing and phase noise reduction techniques," in European Solid-State Circuits Conf., Sept 2011, pp. 491-494.

[18] T. Lee, The Design of CMOS Radio-frequency Integrated Circuits. Cambridge University Press, 1998.

[19] A. Bevilacqua, F. Pavan, C. Sandner, A. Gerosa, and A. Neviani, "A 3.47 GHz Transformer-Based Dual-mode Wideband VCO," in European Solid-State Circuits Conf., Sept 2006, pp. 440-443.

[20] G. Li, L. Liu, Y. Tang, and E. Afshari, "A low-phase-noise wide-tuningrange oscillator based on resonant mode switching," IEEE J. Solid-State Circuits, vol. 47, no. 6, pp. 1295-1308, June 2012.

[21] M. Demirkan, S. Bruss, and R. Spencer, "Design of Wide Tuning-Range CMOS VCOs Using Switched Coupled-Inductors," IEEE J. Solid-State Circuits, vol. 43, no. 5, pp. 1156-1163, May 2008.

[22] E. Mammei, E. Monaco, A. Mazzanti, and F. Svelto, "A 33.6-to$46.2 \mathrm{GHz} 32 \mathrm{~nm}$ CMOS VCO with $177.5 \mathrm{dBc} / \mathrm{Hz}$ minimum noise FOM using inductor splitting for tuning extension," in Int. Solid-State Circuits Conf. Tech. Dig., Feb 2013, pp. 350-351.

[23] J. Yin and H. Luong, "A 57.5-90.1-GHz Magnetically Tuned Multimode CMOS VCO,” IEEE J. Solid-State Circuits, vol. 48, no. 8, pp. 18511861, Aug 2013.

[24] S. Levantino, P. Maffezzoni, F. Pepe, A. Bonfanti, C. Samori, and A. Lacaita, "Efficient calculation of the impulse sensitivity function in oscillators," IEEE Trans. Circuits Syst. II, Exp. Briefs, vol. 59, no. 10, pp. 628-632, Oct 2012.

[25] D. Murphy, J. Rael, and A. Abidi, "Phase Noise in LC Oscillators: A Phasor-Based Analysis of a General Result and of Loaded Q," IEEE Trans. Circuits Syst. I, Reg. Papers, vol. 57, no. 6, pp. 1187-1203, June 2010.

[26] M. Babaie and R. Staszewski, "An Ultra-Low Phase Noise Class-F2 CMOS Oscillator With $191 \mathrm{dBc} / \mathrm{Hz}$ FoM and Long-Term Reliability," IEEE J. Solid-State Circuits, vol. 50, no. 3, pp. 679-692, March 2015.

[27] D. Murphy, Q. Gu, Y.-C. Wu, H.-Y. Jian, Z. Xu, A. Tang, F. Wang, and M.-C. Chang, "A Low Phase Noise, Wideband and Compact CMOS PLL for Use in a Heterodyne 802.15.3c Transceiver," IEEE J. SolidState Circuits, vol. 46, no. 7, pp. 1606-1617, July 2011.

[28] W. Sansen, "1.3 Analog CMOS from 5 micrometer to 5 nanometer," in Int. Solid-State Circuits Conf. Tech. Dig., Feb 2015, pp. 1-6.

[29] D. Zhao and P. Reynaert, "A 60-GHz Dual-Mode Class AB Power Amplifier in 40-nm CMOS," IEEE J. Solid-State Circuits, vol. 48, no. 10, pp. 2323-2337, Oct 2013.

[30] J. Shi, K. Kang, Y. Z. Xiong, J. Brinkhoff, F. Lin, and X.-J. Yuan, "Millimeter-Wave Passives in 45-nm Digital CMOS," IEEE Electron Device Letters, vol. 31, no. 10, pp. 1080-1082, Oct 2010.

[31] B. Razavi, RF Microelectronics, 2nd ed. Upper Saddle River, NJ, USA: Prentice Hall Press, 2011.

[32] Z. Zong, M. Babaie, and R. B. Staszewski, “ 60 GHz 25\% Tuning Range Frequency Generator with Implicit Divider Based on Third Harmonic Extraction with $182 \mathrm{dBc} / \mathrm{Hz}$ FoM," in IEEE Radio Freq. Integr. Circuits Symp. Dig., May 2015, pp. 279-282.

[33] E. Laskin, M. Khanpour, S. Nicolson, A. Tomkins, P. Garcia, A. Cathelin, D. Belot, and S. Voinigescu, "Nanoscale CMOS Transceiver Design in the 90-170-GHz Range," IEEE Trans. Microw. Theory Techn., vol. 57, no. 12, pp. 3477-3490, Dec 2009.

[34] B. Soltanian, H. Ainspan, W. Rhee, D. Friedman, and P. Kinget, "An Ultra-Compact Differentially Tuned 6-GHz CMOS LC-VCO With Dynamic Common-Mode Feedback," IEEE J. Solid-State Circuits, vol. 42, no. 8, pp. 1635-1641, Aug 2007.
[35] A. Mirzaei, M. Mikhemar, and H. Darabi, "A pulling mitigation technique for direct-conversion transmitters," in Int. Solid-State Circuits Conf. Tech. Dig., Feb 2014, pp. 374-375.

[36] B. Razavi, "Design considerations for direct-conversion receivers," IEEE Trans. Circuits Syst. II, Analog Digit. Signal Process.(1993-2003), vol. 44, no. 6, pp. 428-435, Jun 1997.

[37] _ _A study of injection locking and pulling in oscillators," IEEE J. Solid-State Circuits, vol. 39, no. 9, pp. 1415-1424, Sept 2004.

[38] A. Bevilacqua and P. Andreani, "Phase noise analysis of the tunedinput-tuned-output (TITO) oscillator," IEEE Trans. Circuits Syst. II, Exp. Briefs, vol. 59, no. 1, pp. 20-24, Jan 2012.

[39] A. Mazzanti and P. Andreani, "Class-C Harmonic CMOS VCOs, With a General Result on Phase Noise," IEEE J. Solid-State Circuits, vol. 43, no. 12, pp. 2716-2729, Dec 2008.

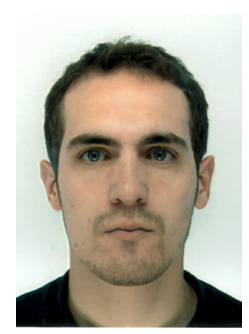

Marco Vigilante (S'14) was born in Carpi, Italy in 1988. He received both the degree of B.S. and M.S. in Electrical Engineering from the Università di Modena, Italy in 2010 and 2012 respectively.

Currently, he is a research assistant at the MICAS laboratories of the $\mathrm{KU}$ Leuven. He is working towards the Ph.D. degree on integrated circuit (IC) design for millimeter-Wave applications.

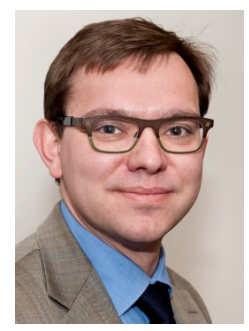

Patrick Reynaert (SM'11) was born in Wilrijk, Belgium, in 1976. He received the Master of Industrial Sciences in Electronics (ing.) from the Karel de Grote Hogeschool, Antwerpen, Belgium in 1998 and both the Master of Electrical Engineering (ir.) and the Ph.D. in Engineering Science (dr.) from the University of Leuven (KU Leuven), Belgium in 2001 and 2006 respectively.

From 2006 to 2007, he was a post-doctoral researcher at UC Berkeley. During the summer of 2007, he was a visiting researcher at Infineon, Villach, Austria. Since October 2007, he is an Associate Professor at the University of Leuven (KU Leuven), department of Electrical Engineering (ESAT) and a staff member of the ESAT-MICAS research group. His main research interests include mm-wave and $\mathrm{THz}$ CMOS circuit design, highspeed circuits and RF power amplifiers.

Dr. Reynaert is the Chair of the IEEE Solid-State Circuits Society Benelux Chapter. He serves or has served on the technical program committees of several international conferences including ISSCC, ESSCIRC, RFIC, IEDM and PRIME. He has served as Associate Editor for IEEE Transactions on Circuits and Systems-I: Regular Papers,and as Guest Editor for the IEEE Journal of Solid-State Circuits. In 2011, he received the TSMC-Europractice Innovation Award. In 2014 he received the Bell Labs Prize (2nd prize). 